\title{
NASAL CARRIAGE OF STAPHYLOCOCCUS AUREUS AND THE QUANTUM OF THEIR METHICILLIN RESISTANCE AMONGST THE HEALTH CARE WORKERS IN A PERIPHERAL TERTIARY CARE CENTRE OF EASTERN INDIA
}

\author{
Partha Sarathi Satpathi ${ }^{1}$, Amit Bikram Maity², Purba Mukherjee ${ }^{3}$, Sanghamitra Satpathi ${ }^{4}$ \\ ${ }^{1}$ Associate Professor, Department of Microbiology, Midnapore Medical College and Hospital, West Bengal, India. \\ ${ }^{2}$ RMO, Department of ENT, Midnapore Medical College and Hospital, West Bengal, India. \\ ${ }^{3}$ Demonstrator, Department of Microbiology, Midnapore Medical College and Hospital, West Bengal, India. \\ ${ }^{4}$ Sr. Dy. Director, Pathology \& I/C IDBU Lab, Ispat General Hospital, Rourlkela, Odisha, India.
}

ABSTRACT: OBJECTIVE: Stapylococcus aureus is an important pathogen causing hospital acquired infection in patients admitted to
different clinical establishments. Amongst them MRSA is most important in causing morbidity, mortality and increase in economic
burden not only to the patients but also to the society at large. Nasal carriage of the organism by the heath care workers always
remains an important source of infection to the hospitalized patients and regular screening of colonized health care workers can
prevent their transmission to the hospital and community. The present study was carried out in search of obtaining the actual level
of nasal carriage in our set up and to arrange a corrective measure in this context. METHOD: A total of 183 nasal swabs were collected from different categories of staffs of Medinipur Medical College and Hospital, Paschim Medinipur, West Bengal from March 2014 to May 2014. Staph aureus isolates were identified by catalase test, coagulase test (Slide and tube) and mannitol fermentation. Methicillin resistance was detected by using cefoxitin disc 30microgram on Mueller Hinton agar with 4\% sodium chloride. Vancomycin resistance was also detected by following CLSI guidelines.

RESULTS: out of the 183 health care workers screened 39(21.7\%) were identified as nasal carrier of Staphylococcus aureus of which $69.23 \%$ are nurses and doctors. Amongst the carriers $12(30.7 \%)$ are colonized by MRSA strains and 27(69.2\%) by MSSA strains. Highest rate nasal colonization by MRSA (75\%) were observed in nurses and doctors combined together.

CONCLUSION: Nasal colonization of Staphylococcus aureus amongst Health care workers, particularly in the most educated group in our set up is quite high and what is more alarming is that $75 \%$ of them are MRSA carriers. They will be the potential source and vehicle of transmission to hospital set up and the community at large. Treatment should be given to them and awareness about hygienic methods are to be developed amongst all categories of HCW. Continuous surveillance will minimize the cost of treatment burden on to the patients and community.

KEYWORDS: Coagulase Negative Staphylococcus Aureus (CONS), Health Care Workers (HCW), Methicillin Resistant Staphylococcus Aureus (MRSA).

HOW TO CITE THIS ARTICLE: Partha Sarathi Satpathi, Amit Bikram Maity, Purba Mukherjee, Sanghamitra Satpathi. "Nasal Carriage of Staphylococcus Aureus and the Quantum of their Methicillin Resistance amongst the Health Care Workers in a Peripheral Tertiary Care Centre of Eastern India". Journal of Evolution of Medical and Dental Sciences 2015; Vol. 4, Issue 90, November 09; Page: 15537-15542, DOI: 10.14260/jemds/2015/2226.

INTRODUCTION: Staphylococcus aureus (S aureus), recognized as an important human pathogen is amongst the clinically most relevant nosocomial pathogen giving rise to increased morbidity, mortality, length of hospital stay and economic burden to the hospitalized patients. ${ }^{1-4}$ In spite of vigorous antibiotic therapy $S$ aureus infections in hospitalized patients have severe consequences ranging from benign superficial skin infections to life threatening infections like Endocarditis, Pneumonia, Meningitis and Septicemia etc. Treatment has become more difficult because of antibiotic resistance as a whole particularly due to Methicillin reaistant $\mathrm{S}$ aureus (MRSA). MRSA has become endemic worldwide within the past two decades. 5 People infected with MRSA are more likely to have longer, more

Financial or Other, Competing Interest: None.

Submission 11-10-2015, Peer Review 12-10-2015,

Acceptance 26-10-2015, Published 07-11-2015.

Corresponding Author:

Dr. Partha Sarathi Satpathi,

Associate Professor,

Department of Microbiology,

Midnapore Medical College and Hospital,

Paschim Medinipur-721101.

E-mail: pssatpathi@gmail.com

DOI:10.14260/jemds/2015/2226. expensive hospital stay and may be more likely to die as a result of the infection. Since its isolation in 1961 MRSA has become a global health problem and a major cause of concern for the infection control management groups.

Approximately $25 \%-30 \%$ of healthy people carry S aureus in their skin or on their nose. Colonization may be either transient or persistent and may be at single or multiple body sites. Although colonization of multiple body sites occurs, anterior nares are the most frequent carriage site. Nasal colonization can be an indicator of high risk for subsequent infection as MRSA is a well-known risk factor whenever Staph aureus colonization is present. ${ }^{6}$ Anterior nares are the major reservoir of S aureus: $20 \%$ of individuals are persistently or asymptomatically colonized, $60 \%$ are intermittently and $20 \%$ are noncarriers. ${ }^{7}$

Health care workers are an important reservoir of $\mathrm{S}$ aureus and several studies has observed the rate of nasal carriage amongst HCW ranging from $16.8 \%$ to $56.1 \%{ }^{8}$ Colonised HCW may develop infection or may remain asymptomatic, but they are a potential source to the patients and can introduce to their families.9-15 Role of health care workers who are MRSA carriers is critical in the transmission of this pathogen not only to the hospitalized patients but also to the community at large. 
Therefore colonization pattern in HCW should be clearly understood for the formulation and establishment of correct infection control strategies in hospital set up.

Prevention of nosocomial infection transmitted by HCW in different clinical establishments can be achieved by the regular screening and eradication of colonized persons. This can give rise to comprehensive infection control policy for this multidrug resistant pathogen. With this background in mind we have undertaken this study to screen the HCW at various levels in our tertiary care set up with the objective to prevent outbreak related to MRSA carriage particularly.

MATERIAL AND METHOD: The study was carried out at Medinipur Medical College and Hospital, Paschim Medinipur for a period of three months from March to May, 2014. Approval from the Institutional Ethical committee and consent from all the staffs were taken to include the in the study. A total of 183 HCWs were included in the screening which comprised of Doctors, Nurse, Attendants and Lab technicians. Variables like age, sex, category, placements in wards, self-reported health status and use of antibiotic in past 30 days were enquired about the participants.

Exclusion criteria were H/O URTI, fever, nasal surgery, smoking, using snuffs, Diabetes, Immunocompromised condition, use of nasal medications and antimicrobial therapy. Nasal swabs were obtained by using sterile cotton swabs moistened with nutrient broth. The swabs were inserted deep into both anterior nares and rotated for five times and immediately transported to the laboratory.

It was inoculated in 5\% sheep blood agar and incubated at $37^{\circ} \mathrm{C}$ for $24 \mathrm{hrs}$. Staph aureus was identified by colony morphology, Gram stain, Catalase test, Coagulase test (both slide and tube) and Mannitol salt fermentation test. Each isolate was then screened for MRSA by showing their susceptibility to Cefoxitin (30microgram) and Oxacillin (1microgram) discs in accordance with the CLSI guidelines. ${ }^{16}$

The susceptibility of the MRSA strains against other antibiotics e.g., Ciprofloxacin, Levofloxacin, Gentamicin, Cefotaxim, Amoxycillin, Azithromycin and Vancomycin was also determined using CLSI guidelines.

Statistical analysis was done by using open epi, version 0.03 $a$ and $p$ value of 0.05 was taken as statistically significant.

RESULTS:In this study a total of 183 Health Care Workers were screened for the nasal carriage of $\mathrm{S}$ aureus in their anterior nares. Out of these, $39(21.47 \%)$ HCWs were detected as nasal carriers of $\mathrm{S}$ aureus and 56 were carrying coagulase negative Staphylococcus whereas 88 samples were negative. Carriage rate in females was $51.28 \%$ whereas in males it was $48.71 \%$ which was statistically significant (table-I). The mean age of the participants in this study was 42 years (age ranges 18-60 years).

The prevalence of nasal carriage of $\mathrm{S}$ aureus in different age groups is $53.84 \%$ in $18-31$ year age group, $30.76 \%$ in $32-45$ yr age group and $15.38 \%$ in $46-60$ yr age group. The carriage rates in different categories of staffs of the hospital are $43.59 \%$ in Nurses, $25.64 \%$ in both Doctors and Laboratory technicians and $5.12 \%$ in the attendants. In ward wise prevalence Medicine tops the list (25.64\%) followed by Orthopedics (23.07\%), Surgery (20.51\%), Pediatrics (17.95\%), G\&O (7.7\%) and ICU (5.12\%)-(table-I).

Out of the total nasal carriers in our study twelve (30.7\%) are MRSA carriers and 27(69.2\%) are MSSA carriers.
In category wise distribution most important MRSA carriers are the Doctors (50\%) followed by Nurses (25\%), Laboratory Technicians (16.66\%) and attendants 8.33\%). Ward wise Medicine and G\&O have 25\% each along with Pediatrics, Orthopedics and Surgery has $16.66 \%$ each. No ICU personnel were identified as MRSA carriers (table-II).

Antibiotic resistance of MRSA strains of Staphylococcus aureus showed $100 \%$ resistance to Cefotaxim and Amoxycillin whereas Ciprofloxacin, Azithromycin, Gentamycin and Levofloxacin showed $91.6 \%, 75 \%$ and $66 \%$ and $58.3 \%$ resistance respectively. Only one strain in our case is observed to be Vancomycin resistant (8.3\%) - (Table-III).

DISCUSSION: Staphylococcus aureus is a frequent cause of nosocomial infections, including bacterimia and wound infections. Approximately $25 \%$ of all nosocomial infections are caused by Staph aureus affecting the patients of both the surgical and nonsurgical wards of different hospitals which leads to increased hospital stay, increased antibiotic use and in this way put an extra economic burden and chance of mortality particularly in underdeveloped or developing countries. ${ }^{17}$

In a developing country such economic burden slows the process of growth. The primary reservoir of Staphylococci is thought to be the vestibulum nasi, though it can be cultured from multiple sites of the skin and mucosal surfaces of carriers. This part of the nose is lined by a fully keratinized epidermis with hairs, sebaceous glands and sweat glands.

The staphylococcal cells can flourish here in the relative absence of human defenses and/or are capable of withstanding the local antibacterial defenses. The bacterial cells need to adhere firmly to prevent their elimination by physicochemical mechanisms. ${ }^{18}$

Out of a total of 183 healthy hospital personnel studied in our case, we have isolated 39 (21.31\%) S aureus and 56(30.6\%) coagulase negative Staphylococcus but 88 of our samples gave negative result. The nasal carriage rate amongst the HCWs in our hospital is $21.31 \%$ which is nearly similar to the studies showed by Citak et al from Turkey $(24.2 \%){ }^{6}$ Golia et al from India. ${ }^{19}(24.84 \%)$ and Nasir et al from Iran. ${ }^{20}$ (26.5\%). Studies by Ahmed et al from Saudi Arabia. ${ }^{21}$ showed a bit higher rate (30.6\%) along with Zermina Rashid et al from Pakistan. ${ }^{22}$ (48\%) whereas a very lower rate of nasal carriage was detected by Khalili et al (12.67\%) from Iran. ${ }^{17}$ and Shakya et al from Nepal. ${ }^{23}$ $(12.5 \%)$.

Nasal carriage rate in females are a bit higher (51.28\%) in our case in comparison to the carriage rate in the case of males $(48.71 \%)$ which is similar to the report by Shakya et al.23 (13.6\% in females and $11.3 \%$ in males). In age-wise distribution of nasal carriage it was found that the young adults (18-31) are the most important reservoir $(53.84 \%)$ of s aureus in our study followed by 32-45 year age group (30.76\%) and 46-60 year age group $(15.38 \%)$. The prevalence of carriage is higher in young individuals was also reported by Ahmad et al.21 (33.4\%) as compared to the aged.

Commonest group of health care workers having nasal carriage are the Nurses (43.59\%) followed by the Doctors (25.64\%), Laboratory technicians $(25.64 \%)$ and the attendants (5.12\%). Overall the group comprising of the Doctors and Nurses (69.23\%) who are supposed to remain in constant touch with the patients in a hospital set up constitutes the largest group of transmitters of infection in our study.

This figure is in agreement with other studies like Ahmad et al. ${ }^{21}$ reported $87.2 \%$ in Doctor and Nurses. 
Zermina Rashid et $\mathrm{al}^{22}$ reported a carriage rate of $66.6 \%$ and $51.8 \%$ respectively in Nurses and Doctors whereas Dimitrov and his coworkers ${ }^{24}$ found $21 \%$ and $14 \%$ nasal carriage in Doctors and Nurses respectively. The organism may be transmitted from the nose to skin where colonization seemed to be more transient. Person to person transmission is the usual mode of spread via hands, nasal discharges and rarely by aerosols. ${ }^{25}$

So maintenance of hand hygiene amongst the Doctors and Nurses is a primary change in habit to be adopted by both. This can prevent the transmission of infections not only to the hospitalized patients but also to their families and community at large. Studying the prevalence of nasal carriage by staffs of different wards it was found that staffs of Medicine are the commonest group (25.64\%) followed by the Orthopedics $(23.07 \%)$, Surgery $(20.51 \%)$, Pediatrics (17.95\%), G\&O (7.7\%) and ICU (5.12\%).

MRSA has been recognized as an important nosocomial pathogen worldwide because of increased rate of multidrug resistant strains it may cause ${ }^{26}$. MRSA is a persistent and ever growing problem for health care Institutions. Minimizing the emergence of this and containing its spreads in both hospital and community remains a great challenge to the health care set up.

This challenge needs to be addressed very seriously by the health care providers of every developing country to minimize the enormous economic burden due to this. Methicillin resistance in S aureus is determined by mec A gene which is coding for the Penicillin binding protein $2 \mathrm{a}$ (PBP2a). Presence of PBP2a makes a susceptible strain resistant to all beta-lactum antibiotics ${ }^{19}$. So methicillin resistance confers resistance to all beta lactum agents. Methicillin resistance may be referred to as an intrinsic means, that the mechanism is chromosomally mediated.

Amongst the nasal carriers of $\mathrm{S}$ aureus in our study $12(30.7 \%)$ were carrying MRSA in their anterior nares and 27(69.2\%) were carrying MSSA. The prevalence of MRSA (31.8\%) observed by Ahmed et al in Saudi Arabia. ${ }^{21}$ is similar to the present study whereas a higher rate $(44.1 \%)$ is observed by a study in Ethiopia. ${ }^{27}$ Lower rate of prevalence was observed by some other studies e.g. Citak et $\mathrm{al}^{6}$ showed $23.6 \%$, Zermina Rashid et al. ${ }^{22} 13.95 \%$ and Laxmi et al. ${ }^{26} 12.2 \%$. Methicillin resistance amongst Staphylococci is present throughout India and it ranges from $27 \%$ in Bombay to $47 \%$ in Bangalore ${ }^{28}$

The present study gives a picture of peripheral tertiary care centers in Eastern India. The MRSA carriage is quite high in our centre indicating the danger of appearance of nosocomial infection in a large section of the patients and also there is a chance of spillage of these strains into the community. The health care workers of the different care facilities should put an extra emphasis on hand hygiene while dealing with the patients to diminish the transmission of MRSA not only to their patients but also to their family members.

Disinfection of hands after each contact with the patient and use of masks, sterile aprons, avoidance of touching nose during work inside hospitals must be performed by all workers in clinical set up to protect patients from nosocomial infection. What is more alarming in the present study is that the highest rate of carriage of MRSA is amongst the doctors (50\%) followed by the Nurses $(25 \%)$. The other groups are technicians $(16.66 \%)$ and attendants $(8.33 \%)$.
So Doctors and Nurses (75\%) are the most potential sources of nosocomial infection in the present study. In other words education level has got no impact on the carriage of MRSA in health care workers. Higher carriage rate of MRSA amongst Doctors and Nurses was observed by Zermina et al. ${ }^{22}$ (18.5\% \& 27.3\%), Ahmed et al. ${ }^{21}$ (All Doctors) and Fadeyi et al. ${ }^{5}$ $(22.7 \%$ \& $16.7 \%)$. In ward wise distribution of nasal carriage of MRSA in the present study it is observed that G\&O and Medicine ward contributes maximum (25\% each) in comparison to others (Orthopedics 16.6\%, Surgery $16.6 \%$ and Pediatrics 16.6\%).

As G\& 0 department also caters patients to the SNCU, NICU and Pediatrics wards, so they can be potential sources of infection to those areas. This in turn will cause increase in mortality, morbidity and increase in cost of treatment. Mupirocin nasal ointment is presently the treatment of choice for decolonization of nasal carriage. It is effective in temporarily eradicating $\mathrm{S}$ aureus from nose. When applied twice daily for five consecutive days it results in elimination rates of $91 \%$ after therapy. ${ }^{29}$

All MRSA strains were subjected to observe the resistance pattern to other antibiotics. The MRSA strains were $100 \%$ resistant to cefotaxime and amoxicillin but $91.6 \%$ to ciprofloxacin, $75 \%$ to Azithromycin, $66 \%$ to Gentamycin, $58.3 \%$ to Levofloxacin. One of the MRSA strain in our study was observed to be resistant to Vancomycin. So the MRSA strains in the present study shows multidrug resistance to regularly used drugs in hospital set up. The reason for this could be injudicious use of regular antibiotics in the hospital and community set up which provides selective pressure favoring resistance to bacterial strains.

Inappropriate use increases the risk for selection and dissemination of antibiotic resistant bacteria. This might be the reason why commonly used inexpensive drugs lead to development of bacterial resistance in developing countries. ${ }^{22}$ Vancomycin resistance to MRSA strains in the present study gives an alarming impression of the status of resistance of even the reserved drugs used for treatment of serious infections in tertiary care centers.

So this drug should not become a regular use drug in present situation. It has to be corrected in a war footing manner to prevent its spread in both hospital and community. An important aspect of our study is that CONS were isolated from anterior nares in a large number (30.6\%) and a big number of them are Methicillin resistant. They are also an important agent of infection in hospital set up and needs surveillance to check their transmission.

To conclude, health care workers in our hospital appears to be a major source of nasal carriage and to prevent nosocomial infection in our set up it needs regular screening and treatment of carriers with mupirocin ointment. There should be a proper infection control rule and regular awareness programs amongst the health care workers.

\section{REFERENCES:}

1. Diekema DJ, et al, Survey of infections due to Staphylococcus species: frequency of occurrence and antimicrobial susceptibility of isolates collected in the United States, Canada, Latin America, Europe, and the Western Pacific region for the SENTRY Antimicrobial Surveillence Program, 1997-1999. Clinical Infectious Diseases 2001; 32 (suppl 2): 114-132. 
2. Kirkland KB, et al. The impact of surgical site infections in the 1990s: attributable mortality, excess length of hospitalization, and extra costs. Infection Control Hospital Epidemiology 1999; 20: 725-730.

3. Kock R, et al. Methicillin resistant Staphylococcus aureus (MRSA): burden of disease and control challenges in Europe. Eurosurveillance 2010; 15:19688.

4. Pittet D, Wengel RP. Nosocomial bloodstream infections. Secular trends in rates, mortality, and contribution to total hospital deaths. Archieves of Internal Medicine 1995; 155: 1177-1184.

5. A Fadeyi, B.O.Bolaji, 0.0.Oyedepo. Methicillin resistant Staphylococcus aureus, carriage among health care workers of critical care units in a Nigerian hospital, American Journal of Infectious Diseases 2010; 6(1): 18-23.

6. Citac S, Bayajit F N and Aksoy F. Nasal carriage and methicillin resistance of Staphylococcus aureus in patients and hospital staff in a tertiary referral center setting. African Journal of Microbiology Research 2011; 5(13):1615-1618.

7. Bruno Gonzalez-Zorn, Jose P. M. Senna, Laurence Fiette, Spencer Shorte, Aurelie Testard, Michel Chignard, Patrice Courvalin, and Catherine GrillotCourvalin. Bacterial and Host Factors Implicated in Nasal Carriage of Methicillin-Resistant Staphylococcus aureus in Mice. Infection and Immunity 2005; 73: 1847-1851.

8. R Goyal, et al. Colonization of methicillin resistant Staphylococcus aureus among healthcare workers in a tertiary care hospital at Delhi. Indian Journal of Medical Sciences 2002; 56(7): 321-324.

9. von Eiff $\mathrm{C}$, et al. Nasal carriage as a source of Staphylococcus aureus bacteremia. Study Group. New England Journal of Medicine 2001; 344: 11-16.

10. Wertheim HF, et al. Risk and outcome of nosocomial Staphylococcus aureus bacteraemia in nasal carriers versus non-carriers. Lancet 2004; 364: 703-705.

11. Blok HE, et al. Role of healthcare workers in outbreaks of methicillin-resistant Staphylococcus aureus: a 10year evaluation from a Dutch university hospital. Infection Control Hospital Epidemiology 2003; 24: 679-685.

12. Tammelin A, et al. Nasal and hand carriage of Staphylococcus aureus in staff at a Department for Thoracic and Cardiovascular Surgery: endogenous or exogenous source? Infection Control Hospital Epidemiology 2003; 24: 686-689.

13. Lu PL, et al. Methicillin-resistant Staphylococcus aureus carriage, infection and transmission in dialysis patients, healthcare workers and their family members. Nephrology Dialysis Transplantation 2008; 23: 1659-1665.

14. Mollema FP, et al. Transmission of methicillin-resistant Staphylococcus aureus to household contacts. Journal of Clinical Microbiology 2010; 48: 202-207.
15. Wagenvoort JH, et al. Risk of re-introduction of methicillinresistant Staphylococcus aureus into the hospital by intrafamilial spread from and to healthcare workers. Journal of Hospital Infection 2005; 59: 67-68.

16. Clinical and Laboratory standard institute. Performance standard for antimicrobial disc susceptibility tests 2012; twentieth supplent. 32 (3): M100-S21.

17. Khalili Md B, Sharifi-Yazdi Md K, Dargahi H and Sadeghian H A. Nasal Colonization rate of Staphylococcus aureus strains among Health Care Service Employee's of Teaching University Hospitals in Yazd. Acta Medica Iranica 2009; 47 (4): 315-317.

18. Kluytmans J, van Beikum A and Verbrugh H. Nasal carriage of staphylococcus aureus: epidemiology, underlying mechanisms and associated risks. Clin Microbiol Rev 1997; 10: 505-20.

19. Golia S, Hittinahalli V, Asha S, Kamath B, Nirmala A R. A study of nasal carriage of MRSA among the health care workers of a tertiary care hospital, Bangalore. International Journal of Basic and Applied Medical Sciences 2013; 3(1): 3-7.

20. Nasiri B, Reza G, and Akbar D. Prevalence of Nasal Carriage of Staphylococcus aureus in Madani Heart Hospital, Tarbiz. Journal of Cardiovascular Research 2010; 2 (3): 13-17.

21. Ahmed S. The prevalence of Staphylococcus aureus colonization among Healthcare Workers at a specialist Hospital in Saudi Arabia. Journal of Clinical and Diagnostic Research 2010; (4):2438-2441.

22. Rashid Z, Farzana K, Sattar A and Murtaza G. Prevalence of nasal Staphylococcus aureus and Methicillin-Resistant Staphylococcus aureus in hospital personnel and associated risk factors. Acta Poloniae Pharmaceutica- Drug Research 2012; 69 (5): 985-991.

23. Shakya B, Shrestha S,Mitra T. Nasal carriage rate of Methicillin resistant Staphylococcus aureus among at National Medical College Teaching Hospital, Birgang, Nepal. Nepal Medical College Journal 2010; 12(1):26-29.

24. Dimitrov Tz, Udo E.E and Grover S. Point surveillance of Staphylococcus aureus carriage among medical staff in Infectious Diseases Hospital. Medical Principles and Practice 2003; 12: 139-144.

25. Working Party Report- Revised guidelines for the control of epidemic Methicillin-resistant Staphylococcus aureus. J Hosp Infect 1990; 16:351-377.

26. Lakshmi S, Kakhandki L.S, Peerapur B.V. Study of nasal carriage of MRSA among the clinical staff and health care workers of a teaching hospital of Karnataka, India. Al Ameen Journal of Medical Science 2012; 5(4): 367-370.

27. Shibabaw A, Abebe T, Mihret A. Nasal carriage rate of methicillin resistant Staphylococcus aureus among Dessie Referral Hospital Health Care Workers; Dessie, Northeast Ethiopia. Antimicrobial Resistance and Infection Control 2013; 2: 25. 
28. Kalyani K, Jayakumar K, and Sunil kumar J. Prevalence of Methicillin-Resistant Staphylococcus aureus among Health Care Workers of Shri Satya Sai Medical College and Hospital- A Tertiary Care Centre. IOSR Journal of Dental and Medical Sciences 2012; 3(2): 23-27.
29. Doebbeling B.N, Breneman D.L, Neu H.C,Ali R, Yangco B.G, Holly H.P, Jr Marsh R.J, Pfaller M.A, McGown JE, and Jr Scully BE. Elimination of Staphylococcus aureus nasal carriage in health care workers: analysis of six clinical trials with calcium mupirocin ointment. Clinical Infectious Diseases 1993; 17: 466-474.

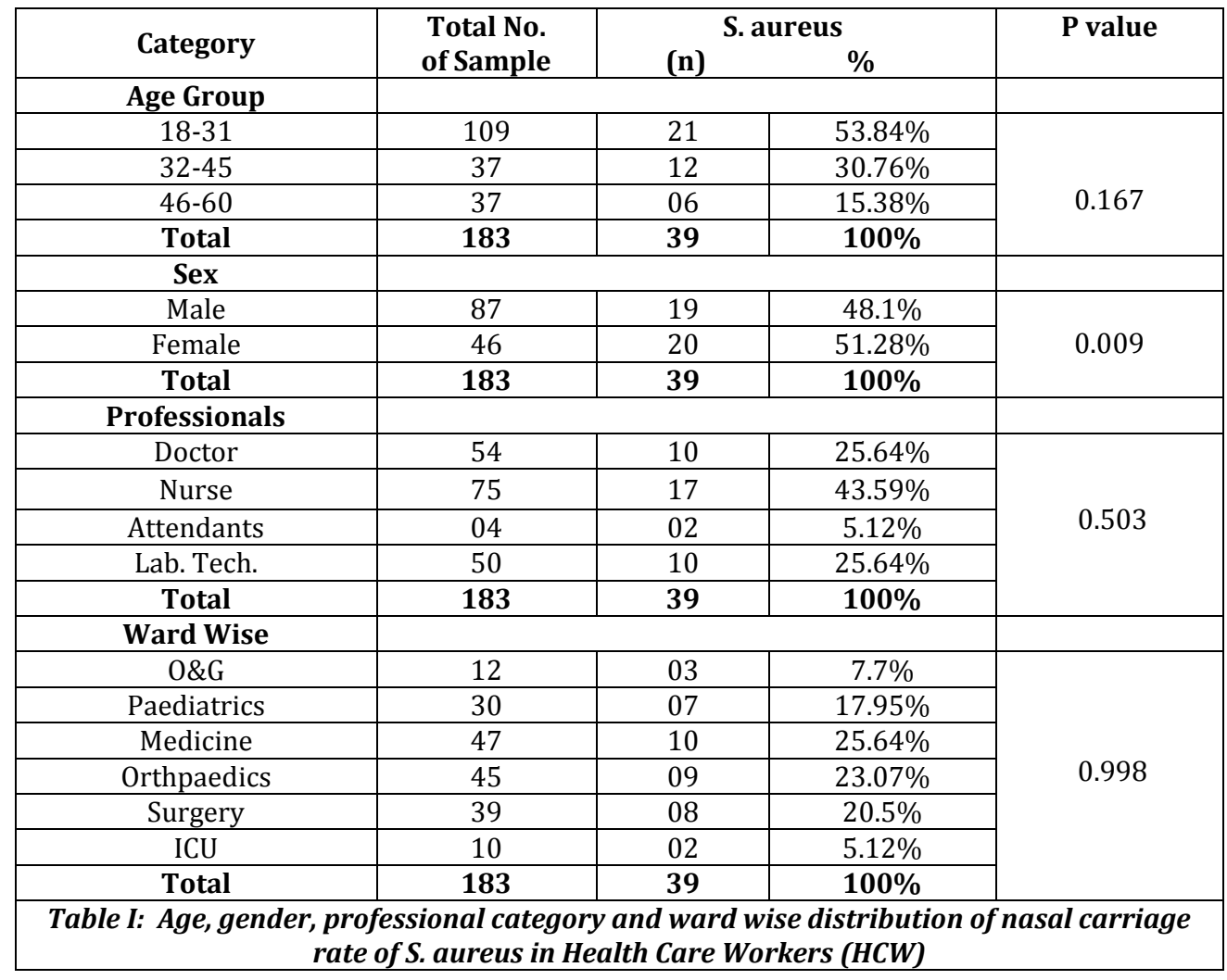

\begin{tabular}{|c|c|c|c|c|}
\hline Group & Total & MRSA (\%) & p value & MSSA (\%) \\
\hline \multicolumn{5}{|c|}{ Category of HCW } \\
\hline Doctor & 54 & $6(50)$ & \multirow{5}{*}{0.145} & $4(14.8)$ \\
\hline Nurse & 75 & $3(25)$ & & $14(51.85)$ \\
\hline Attendants & 04 & $1(8.33)$ & & $1(3.7)$ \\
\hline Lab. Tech. & 50 & $2(16.66)$ & & $8(29.63)$ \\
\hline Total & 183 & 12 & & 27 \\
\hline \multicolumn{5}{|l|}{ Ward Wise } \\
\hline O\&G & 12 & $3(25)$ & \multirow{7}{*}{0.166} & Nil \\
\hline Pediatrics & 30 & $2(16.66)$ & & $5(18.51)$ \\
\hline Medicine & 47 & $3(25)$ & & $7(25.92)$ \\
\hline Orthopedics & 45 & $2(16.66)$ & & $7(25.92)$ \\
\hline Surgery & 39 & $2(16.66)$ & & $6(22.22)$ \\
\hline ICU & 10 & Nil & & $2(7.4)$ \\
\hline Total & 183 & 12 & & 27 \\
\hline
\end{tabular}




\begin{tabular}{|c|c|c|}
\hline Sl. No. & Antibiotic & Resistant MRSA Strains (n=12) \\
\hline 1 & Cefotaxime & $12(100 \%)$ \\
\hline 2 & Amoxycillin & $12(100 \%)$ \\
\hline 3 & Gentamycin & $8(66 \%)$ \\
\hline 4 & Azithromycin & $9(75 \%)$ \\
\hline 5 & Ciprofloxacin & $11(91.6 \%)$ \\
\hline 6 & Levofloxacin & $158.3 \%)$ \\
\hline 7 & Vancomycin & $1(8.3 \%)$ \\
\hline \multicolumn{2}{|c|}{ Table III: Antibiotic resistance pattern of MRSA strains among Health Care Workers } \\
\hline
\end{tabular}

